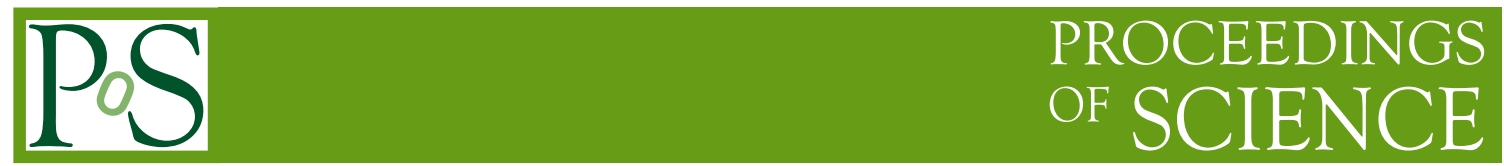

\title{
The S-Matrix of AdS/CFT and Yangian Symmetry
}

\author{
Niklas Beisert* \\ Max-Planck-Institut für Gravitationsphysik \\ Albert-Einstein-Institut \\ Am Mühlenberg 1, 14476 Potsdam, Germany \\ E-mail: nbeiserteaei.mpg.de
}

We review the algebraic construction of the S-matrix of AdS/CFT. We also present its symmetry algebra which turns out to be a Yangian of the centrally extended $\mathfrak{s u}(2 \mid 2)$ superalgebra.

Bethe Ansatz: 75 years later

October 19-21 2006,

Brussels, Belgium

\footnotetext{
* Speaker.
} 


\section{Introduction and Overview}

Bethe's ansatz [1] for solving a one-dimensional integrable model was and remains a powerful tool in contemporary theoretical physics: 75 years ago it solved one of the first models of quantum mechanics, the Heisenberg spin chain [2]; today it provides exact solutions for the spectra of certain gauge and string theories and thus helps us understand their duality [3] better. Since the discovery of integrable structures in planar $\mathscr{N}=4$ supersymmetric gauge theory [4-6] and in planar IIB string theory on $A d S_{5} \times S^{5}[7,8]$ the tools for computing and comparing the spectra of both models have evolved rapidly. We now have complete asymptotic Bethe equations $[9,10]$ which interpolate smoothly between the perturbative regimes in gauge and string theory and which agree with all available data.

In this note we will focus on the S-matrix [11] in the excitation picture above a ferromagnetic ground state. We start by reviewing the algebraic construction of the S-matrix in Sec. 2. In Sec. 3 we subsequently show that this S-matrix has indeed a larger symmetry algebra: a Yangian.

\section{The Universal Enveloping Algebra $U\left(\mathfrak{s u}(2 \mid 2) \ltimes \mathbb{R}^{2}\right)$}

In this section the results on the S-matrix of AdS/CFT shall be reviewed from an algebraic point of view. The applicable symmetry is a central extension $\mathfrak{h}$ of the Lie superalgebra $\mathfrak{s u}(2 \mid 2)$ which we consider first. We continue by presenting the Hopf algebra structure of its universal enveloping algebra and its fundamental representation. Finally, we comment on the S-matrix and its dressing phase factor.

Lie Superalgebra. The symmetry in the excitation picture for light cone string theory on $A d S_{5} \times$ $S^{5}$ and for single-trace local operators in $\mathscr{N}=4$ supersymmetric gauge theory is given by two copies of the Lie superalgebra [12, 13]

$$
\mathfrak{h}:=\mathfrak{s u}(2 \mid 2) \ltimes \mathbb{R}^{2}=\mathfrak{p s u}(2 \mid 2) \ltimes \mathbb{R}^{3} .
$$

It is a central extension of the standard Lie superalgebras $\mathfrak{s u}(2 \mid 2)$ or $\mathfrak{p s u}(2 \mid 2)$, see [14]. It is generated by the $\mathfrak{s u}(2) \times \mathfrak{s u}(2)$ generators $\mathfrak{R}_{b}^{a}, \mathfrak{L}^{\alpha}{ }_{\beta}$, the supercharges $\mathfrak{Q}^{\alpha}{ }_{b}, \mathfrak{S}^{a}{ }_{\beta}$ and the central charges $\mathfrak{C}, \mathfrak{P}, \mathfrak{K}$. The Lie brackets of the $\mathfrak{s u}(2)$ generators take the standard form

$$
\begin{array}{lll}
{\left[\mathfrak{R}^{a}{ }_{b}, \mathfrak{R}^{c}{ }_{d}\right]=\delta_{b}^{c} \mathfrak{R}^{a}{ }_{d}-\delta_{d}^{a} \mathfrak{R}^{c}{ }_{b},} & {\left[\mathfrak{L}^{\alpha}{ }_{\beta}, \mathfrak{L}^{\gamma}{ }_{\delta}\right]=\delta_{\beta}^{\gamma} \mathfrak{L}^{\alpha}{ }_{\delta}-\delta_{\delta}^{\alpha} \mathfrak{L}_{\beta}^{\gamma},} \\
{\left[\mathfrak{R}^{a}{ }_{b}, \mathfrak{Q}_{d}^{\gamma}\right]=-\delta_{d}^{a} \mathfrak{Q}_{b}^{\gamma}+\frac{1}{2} \delta_{b}^{a} \mathfrak{Q}_{d}^{\gamma},} & {\left[\mathfrak{L}^{\alpha}{ }_{\beta}, \mathfrak{Q}^{\gamma}{ }_{d}\right]=+\delta_{\beta}^{\gamma} \mathfrak{Q}^{\alpha}{ }_{d}-\frac{1}{2} \delta_{\beta}^{\alpha} \mathfrak{Q}^{\gamma}{ }_{d},} \\
{\left[\mathfrak{R}^{a}{ }_{b}, \mathfrak{S}^{c}{ }_{\delta}\right]=+\delta_{b}^{c} \mathfrak{S}^{a}{ }_{\delta}-\frac{1}{2} \delta_{b}^{a} \mathfrak{S}^{c}{ }_{\delta},} & {\left[\mathfrak{L}^{\alpha}{ }_{\beta}, \mathfrak{S}^{c}{ }_{\delta}\right]=-\delta_{\delta}^{\alpha} \mathfrak{S}^{c}{ }_{\beta}+\frac{1}{2} \delta_{\beta}^{\alpha} \mathfrak{S}^{c}{ }_{\delta} .}
\end{array}
$$

The Lie brackets of two supercharges yield

$$
\begin{aligned}
& \left\{\mathfrak{Q}_{b}^{\alpha}, \mathfrak{S}^{c}{ }_{\delta}\right\}=\delta_{b}^{c} \mathfrak{L}^{\alpha}{ }_{\delta}+\delta_{\delta}^{\alpha} \mathfrak{R}_{b}^{c}+\delta_{b}^{c} \delta_{\delta}^{\alpha} \mathfrak{C}, \\
& \left\{\mathfrak{Q}^{\alpha}{ }_{b}, \mathfrak{Q}^{\gamma}{ }_{d}\right\}=\varepsilon^{\alpha \gamma} \varepsilon_{b d} \mathfrak{P}, \\
& \left\{\mathfrak{S}^{a}{ }_{\beta}, \mathfrak{S}^{c}{ }_{\delta}\right\}=\varepsilon^{a c} \varepsilon_{\beta \delta} \mathfrak{K} .
\end{aligned}
$$

The remaining Lie brackets vanish. 


$$
\begin{aligned}
\Delta \mathfrak{R}^{a}{ }_{b} & =\mathfrak{R}^{a}{ }_{b} \otimes 1+1 \otimes \mathfrak{R}^{a}{ }_{b}, \\
\Delta \mathfrak{L}^{\alpha}{ }_{\beta} & =\mathfrak{L}^{\alpha}{ }_{\beta} \otimes 1+1 \otimes \mathfrak{L}^{\alpha}{ }_{\beta}, \\
\Delta \mathfrak{Q}^{\alpha}{ }_{b} & =\mathfrak{Q}^{\alpha}{ }_{b} \otimes 1+\mathscr{U}^{+1} \otimes \mathfrak{Q}^{\alpha}{ }_{b}, \\
\Delta \mathfrak{S}^{a}{ }_{\beta} & =\mathfrak{S}^{a}{ }_{\beta} \otimes 1+\mathscr{U}^{-1} \otimes \mathfrak{S}^{a}{ }_{\beta}, \\
\Delta \mathfrak{C} & =\mathfrak{C} \otimes 1+1 \otimes \mathfrak{C}, \\
\Delta \mathfrak{P} & =\mathfrak{P} \otimes 1+\mathscr{U}^{+2} \otimes \mathfrak{P}, \\
\Delta \mathfrak{K} & =\mathfrak{K} \otimes 1+\mathscr{U}^{-2} \otimes \mathfrak{K}, \\
\Delta \mathscr{U} & =\mathscr{U} \otimes \mathscr{U} .
\end{aligned}
$$

Table 1: The coproduct of the braided universal enveloping algebra $U(\mathfrak{h})$.

Where appropriate, we shall use the collective symbol $\mathfrak{J}^{A}$ for the generators. The Lie brackets then take the standard form

$$
\left[\mathfrak{J}^{A}, \mathfrak{J}^{B}\right]=f_{C}^{A B} \mathfrak{J}^{C} .
$$

For simplicity of notation, we shall pretend that all generators are bosonic; the generalisation to fermionic generators by insertion of suitable signs and graded commutators is straightforward.

Hopf Algebra. Next we consider the universal enveloping algebra $U(\mathfrak{h})$ of $\mathfrak{h}$. The construction of the product is standard, and one identifies the Lie brackets (2.4) with graded commutators. For the coproduct one can introduce a non-trivial braiding $[15,16]$

$$
\Delta \mathfrak{J}^{A}=\mathfrak{J}^{A} \otimes 1+\mathscr{U}^{[A]} \otimes \mathfrak{J}^{A}
$$

with some abelian ${ }^{1}$ generator $\mathscr{U}$ (a priori unrelated to the algebra) and the grading

$$
[\mathfrak{R}]=[\mathfrak{L}]=[\mathfrak{C}]=0, \quad[\mathfrak{Q}]=+1, \quad[\mathfrak{S}]=-1, \quad[\mathfrak{P}]=+2, \quad[\mathfrak{K}]=-2 .
$$

The coproduct is spelt out in Tab. 1 for the individual generators. The above grading is derived from the Cartan charge of the $\mathfrak{s l}(2)$ automorphism [14] of the algebra $\mathfrak{h}$ and therefore the coproduct is compatible with the algebra relations.

We should define the remaining structures of the Hopf algebra: the antipode $\mathrm{S}$ and the counit $\varepsilon[15,16]$. The antipode is an anti-homomorphism which acts on the generators as

$$
\mathrm{S}(1)=1, \quad \mathrm{~S}(\mathscr{U})=\mathscr{U}^{-1}, \quad \mathrm{~S}\left(\mathfrak{J}^{A}\right)=-\mathscr{U}^{-[A]} \mathfrak{J}^{A} .
$$

The counit acts non-trivially only on 1 and $\mathscr{U}$

$$
\varepsilon(1)=\varepsilon(\mathscr{U})=1, \quad \varepsilon\left(\mathfrak{J}^{A}\right)=0 .
$$

\footnotetext{
${ }^{1}$ Curiously, we can include the supersymmetric grading $(-1)^{\mathscr{F}}$ in the generator $\mathscr{U}$ to manually impose the correct statistics. This is helpful for an implementation within a computer algebra system. In this case $\mathscr{U}$ would anticommute with fermionic generators.
} 
Cocommutativity. This coproduct is in general not quasi-cocommutative as can easily be seen by considering the central charges $\mathfrak{P}, \mathfrak{K}$ in Tab. 1 . To make it quasi-cocommutative we have to satisfy the constraints [15]

$$
\mathfrak{P} \otimes\left(1-\mathscr{U}^{+2}\right)=\left(1-\mathscr{U}^{+2}\right) \otimes \mathfrak{P}, \quad \mathfrak{K} \otimes\left(1-\mathscr{U}^{-2}\right)=\left(1-\mathscr{U}^{-2}\right) \otimes \mathfrak{K} .
$$

They are solved by identifying the central charges $\mathfrak{P}, \mathfrak{K}$ with the braiding factor $\mathscr{U}$ as follows [16]

$$
\mathfrak{P}=g \alpha\left(1-\mathscr{U}^{+2}\right), \quad \mathfrak{K}=g \alpha^{-1}\left(1-\mathscr{U}^{-2}\right) .
$$

This leads to the following quadratic constraint

$$
\mathfrak{P} \mathfrak{K}-g \alpha^{-1} \mathfrak{P}-g \alpha \mathfrak{K}=0 .
$$

It was furthermore shown in [17] that the coproduct is quasi-triangular, at least at the level of central charges, see also [18].

Fundamental Representation. The algebra $\mathfrak{h}$ has a four-dimensional representation [13] which we will call fundamental. The corresponding multiplet has two bosonic states $\left|\phi^{a}\right\rangle$ and two fermionic states $\left|\psi^{\alpha}\right\rangle$. The action of the two sets of $\mathfrak{s u}(2)$ generators has to be canonical

$$
\begin{aligned}
& \mathfrak{R}_{b}^{a}\left|\phi^{c}\right\rangle=\delta_{b}^{c}\left|\phi^{a}\right\rangle-\frac{1}{2} \delta_{b}^{a}\left|\phi^{c}\right\rangle, \\
& \mathfrak{L}^{\alpha}{ }_{\beta}\left|\psi^{\gamma}\right\rangle=\delta_{\beta}^{\gamma}\left|\psi^{\alpha}\right\rangle-\frac{1}{2} \delta_{\beta}^{\alpha}\left|\psi^{\gamma}\right\rangle .
\end{aligned}
$$

The supersymmetry generators must also act in a manifestly $\mathfrak{s u}(2) \times \mathfrak{s u}(2)$ covariant way

$$
\begin{aligned}
\mathfrak{Q}^{\alpha}{ }_{a}\left|\phi^{b}\right\rangle & =a \delta_{a}^{b}\left|\psi^{\alpha}\right\rangle, \\
\mathfrak{Q}^{\alpha}{ }_{a}\left|\psi^{\beta}\right\rangle & =b \varepsilon^{\alpha \beta} \varepsilon_{a b}\left|\phi^{b}\right\rangle, \\
\mathfrak{S}^{a}{ }_{\alpha}\left|\phi^{b}\right\rangle & =c \varepsilon^{a b} \varepsilon_{\alpha \beta}\left|\psi^{\beta}\right\rangle, \\
\mathfrak{S}^{a}{ }_{\alpha}\left|\psi^{\beta}\right\rangle & =d \delta_{\alpha}^{\beta}\left|\phi^{a}\right\rangle .
\end{aligned}
$$

We can write the four parameters $a, b, c, d$ using the parameters $x^{ \pm}, \gamma$ and the constants $g, \alpha$ as

$$
a=\sqrt{g} \gamma, \quad b=\sqrt{g} \frac{\alpha}{\gamma}\left(1-\frac{x^{+}}{x^{-}}\right), \quad c=\sqrt{g} \frac{i \gamma}{\alpha x^{+}}, \quad d=\sqrt{g} \frac{x^{+}}{i \gamma}\left(1-\frac{x^{-}}{x^{+}}\right) .
$$

The parameters $x^{ \pm}$(together with $\gamma$ ) label the representation and they must obey the constraint

$$
x^{+}+\frac{1}{x^{+}}-x^{-}-\frac{1}{x^{-}}=\frac{i}{g} .
$$

The three central charges $\mathfrak{C}, \mathfrak{P}, \mathfrak{K}$ and $\mathscr{U}$ are represented by the values $C, P, K$ and $U$ which read

$$
C=\frac{1}{2} \frac{1+1 / x^{+} x^{-}}{1-1 / x^{+} x^{-}}, \quad P=g \alpha\left(1-\frac{x^{+}}{x^{-}}\right), \quad K=\frac{g}{\alpha}\left(1-\frac{x^{-}}{x^{+}}\right), \quad U=\sqrt{\frac{x^{+}}{x^{-}}} .
$$

They furthermore obey the quadratic relation $C^{2}-P K=\frac{1}{4}$. Note that the corresponding quadratic combination of central charges $\mathfrak{C}^{2}-\mathfrak{P} \mathfrak{K}$ is singled out by being invariant under the $\mathfrak{s l}(2)$ external automorphism. 
Fundamental S-Matrix. In $[13,17]$ an S-matrix acting on the tensor product of two fundamental representations was derived. It was constructed by imposing invariance under the algebra $\mathfrak{h}$

$$
\left[\Delta \mathfrak{J}^{A}, \mathscr{S}\right]=0
$$

We will not reproduce the result here, it is given in [17]. Note that we have to fix the parameters $\xi=U=\sqrt{x^{+} / x^{-}}$in order to make the action of the generators compatible with the coproduct $(2.5)^{2}$

This S-matrix has several interesting properties. Firstly, it is not of difference form; it cannot be written as a function of the difference of some spectral parameters. Secondly, the S-matrix could be determined uniquely up to one overall function merely by imposing a Lie-type symmetry (2.17) [13]. This unusual fact is related to an unusual feature of representation theory of the algebra $\mathfrak{h}$ : The tensor product of two fundamental representations is irreducible in almost all cases [17].

Intriguingly this S-matrix is equivalent to Shastry's R-matrix [21] of the one-dimensional Hubbard model [22]. Furthermore the Bethe equations [13] contain two copies of the Lieb-Wu equations for the Hubbard model [23]. These observations of [17] establish a link between an important model of condensed matter physics and string theory (complementary to the one in [24]).

Finally, let us note that one can derive (asymptotic) Bethe equations from the S-matrix and thus confirm the conjecture in [9]. So far this step has been performed in two different ways: by means of the nested coordinate [13] and the algebraic [20] Bethe ansatz.

Phase Factor. The remaining overall phase factor of the S-matrix clearly cannot be determined by demanding invariance under $\mathfrak{h}$. The phase factor was computed to some approximation from gauge theory [25] and from string theory [26-29]. The problem of an algebraically undetermined phase factor is in fact generic. Usually one imposes a further crossing symmetry relation to obtain a constraint on it. Indeed the known string phase factor is consistent with crossing symmetry [30] as was shown in [31]. By substituting a suitable ansatz [32] for the phase factor into the crossing symmetry relation a conjecture for the all-orders phase factor at strong coupling was made in [33].

A corresponding all-orders expansion at weak coupling was presented in [10]. The latter conjecture was obtained by a sort of analytic continuation in the perturbative order of the series. Let us illustrate this principle by means of a very simple example: Consider the rational function $f(x)=1 /(1-x)$. It has the following expansions at $x=0$ and at $x=\infty$

$$
f(x) \stackrel{x \rightarrow 0}{=} \sum_{n=0}^{\infty} a_{n} x^{n}, \quad f(x) \stackrel{x \rightarrow \infty}{=} \sum_{n=1}^{\infty} b_{n} x^{-n}
$$

with $a_{n}=1$ and $b_{n}=-1$. When we consider $a_{n}$ and $b_{n}$ as analytic functions of the index, we can make the observation ("reciprocity")

$$
a_{n}=-b_{-n} .
$$

\footnotetext{
${ }^{2}$ This identification removes all braiding factors from the S-matrix in [17] which will thus satisfy the standard Yang-Baxter (matrix) equation, see also [13, 19, 20].
} 
Of course there are various ways in which the two functions +1 and -1 could be related, but the choice (2.19) appears to work for a surprisingly large class of functions! $!^{3}$ It was proved in [36] that it does apply for the conjectured expansion of the phase factor. Very useful integral expressions for the phase have recently appeared in $[37,38]$. The dressing phase can also be obtained as an effective quantity [39] (see also [40]) from the scattering mediated by a non-trivial vacuum state [41, 24, 42]. While this is certainly encouraging in general, it is at the same time strange from the Hopf algebra point of view to use an S-matrix which does not obey the crossing relation [39, 40]. This calls for further investigations.

Several tests of the phase have recently appeared, they are based on four-loop unitary scattering methods [43], numerical evaluation [44, 45], analytic methods [44, 36, 46, 47] and on taking a certain highly non-trivial limit [48].

\section{The Yangian $Y\left(\mathfrak{s u}(2 \mid 2) \ltimes \mathbb{R}^{2}\right)$}

In the section we investigate Yangian symmetry $[49,50]$ for the above S-matrix. We will start with a very brief review of Yangian symmetry for generic S-matrices (see [51, 52] for more extensive reviews), and then we apply the framework to the S-matrix discussed above.

Yangians and S-Matrices. Typically the symmetries of rational S-matrices are of Yangian type. The Yangian $Y(\mathfrak{g})$ of a Lie algebra $\mathfrak{g}$ is a deformation of the universal enveloping algebra of half the affine extension of $\mathfrak{g}$.

More plainly, it is generated by the g-generators $\mathfrak{J}^{A}$ and the Yangian generators $\widehat{\mathfrak{J}}^{A}$. Their commutators take the generic form

$$
\begin{aligned}
& {\left[\mathfrak{J}^{A}, \mathfrak{J}^{B}\right]=f_{C}^{A B} \mathfrak{J}^{C},} \\
& {\left[\mathfrak{J}^{A}, \widehat{\mathfrak{J}}^{B}\right]=f_{C}^{A B} \widehat{\mathfrak{J}}^{C},}
\end{aligned}
$$

and they should obey the Jacobi and Serre relations

$$
\begin{aligned}
& {\left[\mathfrak{J}^{[A},\left[\mathfrak{J}^{B}, \mathfrak{J}^{C]}\right]\right]=0} \\
& {\left[\mathfrak{J}^{[A},\left[\mathfrak{J}^{B}, \widehat{\mathfrak{J}}^{C]}\right]\right]=0} \\
& {\left[\widehat{\mathfrak{J}}^{[A},\left[\widehat{\mathfrak{J}}^{B}, \mathfrak{J}^{C]}\right]\right]=\frac{1}{4} \hbar^{2} f_{D}^{A G} f_{E}^{B H} f_{F}^{C K} f_{G H K} \mathfrak{J}^{\{D} \mathfrak{J}^{E} \mathfrak{J}^{F\}} .}
\end{aligned}
$$

The symbol $f_{A B C}=g_{A D} g_{B E} f_{C}^{D E}$ represents the structure constants $f_{C}^{A D}$ with two indices lowered by means of the inverse of the Cartan-Killing forms $g_{A D}$ and $g_{B E}$. The brackets \{\} and [ ] at the level of indices imply total symmetrisation and anti-symmetrisation, respectively. Finally, $\hbar$ is a scale parameter whose value plays no physical role. The first two relations lead to a constraint on the structure constants $f_{C}^{A B}$. The third relation ${ }^{4}$ is a deformation of the Serre relation for affine extensions of Lie algebras.

\footnotetext{
${ }^{3}$ Among other physical examples, we have identified circular Maldacena-Wilson loops [34] and non-critical string theory [35] where this reciprocity can be applied. Furthermore, summation by the Euler-MacLaurin formula (also known as zeta-function regularisation) is consistent with it. I thank Curt Callan, Marcos Mariño and Tristan McLoughlin for discussions of this principle.

${ }^{4}$ For $\mathfrak{g}=\mathfrak{s u}(2)$ it has to be replaced by a quartic relation.
} 
The Yangian is a Hopf algebra and the coproduct takes the standard form

$$
\begin{aligned}
\Delta \mathfrak{J}^{A} & =\mathfrak{J}^{A} \otimes 1+1 \otimes \mathfrak{J}^{A} \\
\Delta \widehat{\mathfrak{J}}^{A} & =\widehat{\mathfrak{J}}^{A} \otimes 1+1 \otimes \widehat{\mathfrak{J}}^{A}+\frac{1}{2} \hbar f_{B C}^{A} \mathfrak{J}^{B} \otimes \mathfrak{J}^{C}
\end{aligned}
$$

where $f_{B C}^{A}=g_{B D} f_{C}^{A D}$. The antipode $\mathrm{S}$ is defined by

$$
\mathrm{S}\left(\mathfrak{J}^{A}\right)=-\mathfrak{J}^{A}, \quad \mathrm{~S}\left(\widehat{\mathfrak{J}}^{A}\right)=-\widehat{\mathfrak{J}}^{A}+\frac{1}{4} \hbar f_{B C}^{A} f_{D}^{B C} \mathfrak{J}^{D}
$$

and the counit $\varepsilon$ takes the standard form

$$
\varepsilon(1)=1, \quad \varepsilon\left(\mathfrak{J}^{A}\right)=\varepsilon\left(\widehat{\mathfrak{J}}^{A}\right)=0 .
$$

For the study of integrable systems, the evaluation representations of the Yangian are of special interest. For these the action of the Yangian generators $\widehat{\mathfrak{J}}^{A}$ is proportional to the Lie generators

$$
\widehat{\mathfrak{J}}^{A}|u\rangle=\hbar u \mathfrak{J}^{A}|u\rangle
$$

Here $|u\rangle$ is some state of the evaluation module with spectral parameter $u$. This Yangian representation is finite-dimensional if the $\mathfrak{g}$-representation is. One merely has to ensure that the Serre relation (3.2) is satisfied. This is indeed not the case for all representations of all Lie algebras. The power of the Yangian symmetry lies in the fact that tensor products of evaluation representations are typically irreducible (except for special values of their spectral parameters). This allows for simple proofs (e.g. for the Yang-Baxter relation) by representation theory arguments.

Let us finally consider the connection to the S-matrix. The S-matrix is a permutation operator; it acts by interchanging two modules of the algebra

$$
\mathscr{S}: \mathbb{V}_{1} \otimes \mathbb{V}_{2} \rightarrow \mathbb{V}_{2} \otimes \mathbb{V}_{1}
$$

In particular, for the tensor product of two evaluation modules one has

$$
\mathscr{S}\left|u_{1}, u_{2}\right\rangle \sim\left|u_{2}, u_{1}\right\rangle .
$$

Invariance of the S-matrix under the Yangian means

$$
\left[\Delta \mathfrak{J}^{A}, \mathscr{S}\right]=\left[\Delta \widehat{\mathfrak{J}}^{A}, \mathscr{S}\right]=0
$$

for all generators $\mathfrak{J}^{A}, \widehat{\mathfrak{J}}^{A}$. The existence of such an S-matrix is equivalent to quasi-cocommutativity of $Y(\mathfrak{g})$. Note that only the difference of spectral parameters appears in the invariance condition: We can write the action of the coproduct of Yangian generators on the evaluation module $\left|u_{1}, u_{2}\right\rangle$ as

$$
\Delta \widehat{\mathfrak{J}}^{A} \simeq\left(u_{1}-u_{2}\right) \mathfrak{J}^{A} \otimes 1+u_{2} \Delta \mathfrak{J}^{A}+\hbar f_{B C}^{A} \mathfrak{J}^{B} \otimes \mathfrak{J}^{C} .
$$

Here the first equation in (3.9) ensures that the term proportional to $u_{2}$ drops out from the second equation. Therefore the S-matrix typically depends on the difference $u_{1}-u_{2}$ of spectral parameters only. 
Yangians in AdS/CFT. Yangian symmetries for planar AdS/CFT have been investigated in [53], both for classical string theory and for gauge theory at leading order, see also [54-56] Yangian symmetry also persists to higher perturbative orders in both models [25, 57-60] and it is likely that it also exists at finite coupling. This Yangian can be understood as a symmetry of the Hamiltonian on an infinite world sheet or as an expansion of the full monodromy matrix. The Lie symmetry in this picture is $\mathfrak{p s u}(2,2 \mid 4)$ and the Yangian would be $\mathrm{Y}(\mathfrak{p} \mathfrak{s u}(2,2 \mid 4))$.

Here we consider a different picture of well-separated excitations on a ferromagnetic ground state and of their scattering matrix. In this picture the Lie symmetry reduces to two copies of $\mathfrak{h}$ and the corresponding Yangian would be $\mathrm{Y}(\mathfrak{h})$. Our Yangian should arise as a subalgebra of the full Yangian $\mathrm{Y}(\mathfrak{p} \mathfrak{s u}(2,2 \mid 4))$ when acting on asymptotic excitation states.

Hopf Algebra. Let us now consider $\mathrm{Y}(\mathfrak{h})$. We have already studied the universal enveloping algebra $U(\mathfrak{h})$. All we still need to do is to introduce one generator $\widehat{\mathfrak{J}}^{A}$ for each $\mathfrak{J}^{A}$ obeying the relations $(3.1,3.2)$, and define its coproduct, antipode as well as counit.

In (2.5) we have defined a braided coproduct for the universal enveloping algebra. For consistency with the Serre relations, we also have to apply an analogous braiding to the standard Yangian coproduct

$$
\Delta \widehat{\mathfrak{J}}^{A}=\widehat{\mathfrak{J}}^{A} \otimes 1+\mathscr{U}^{[A]} \otimes \widehat{\mathfrak{J}}^{A}+\hbar f_{B C}^{A} \mathfrak{J}^{B} \mathscr{U}^{[C]} \otimes \mathfrak{J}^{C} .
$$

Note that lowering an index requires to use the inverse Cartan-Killing form of the algebra. In the case of $\mathfrak{h}$ the Cartan-Killing form is degenerate and we need to extend the algebra by the $\mathfrak{s l}(2)$ outer automorphism, see [17]. Effectively, lowering an index leads to an interchange of the automorphism generators with the central charges. We refrain from spelling out the Cartan-Killing form or the modified structure constants. Instead we present the complete set of coproducts of Yangian generators in Tab. 2, where we also fix the value of $\hbar$.

For the sake of completeness we state the antipode ${ }^{5}$ and the counit

$$
\mathrm{S}\left(\widehat{\mathfrak{J}}^{A}\right)=-\mathscr{U}^{-[A]} \widehat{\mathfrak{J}}^{A}, \quad \varepsilon\left(\widehat{\mathfrak{J}}^{A}\right)=0 .
$$

Cocommutativity. An important question is if this coproduct can be quasi-cocommutative. ${ }^{6} \mathrm{~A}$ first step is to consider the central generators $\widehat{\mathfrak{C}}, \widehat{\mathfrak{P}}, \widehat{\mathfrak{K}}$. For that purpose it is favourable to choose suitable combinations

$$
\begin{aligned}
\widehat{\mathfrak{C}}^{\prime} & =\widehat{\mathfrak{C}}+g \alpha^{-1} \mathfrak{P}-g \alpha \mathfrak{K}, \\
\widehat{\mathfrak{P}}^{\prime} & =\widehat{\mathfrak{P}}+\mathfrak{C}(\mathfrak{P}-2 g \alpha), \\
\widehat{\mathfrak{K}}^{\prime} & =\widehat{\mathfrak{K}}-\mathfrak{C}\left(\mathfrak{K}-2 g \alpha^{-1}\right),
\end{aligned}
$$

for whom ${ }^{7}$ the coproduct almost trivialises

$$
\begin{aligned}
\Delta \widehat{\mathfrak{C}}^{\prime} & =\widehat{\mathfrak{C}}^{\prime} \otimes 1+1 \otimes \widehat{\mathfrak{C}}^{\prime}, \\
\Delta \widehat{\mathfrak{P}}^{\prime} & =\widehat{\mathfrak{P}}^{\prime} \otimes 1+\mathscr{U}^{+2} \otimes \widehat{\mathfrak{P}}^{\prime}, \\
\Delta \widehat{\mathfrak{K}}^{\prime} & =\widehat{\mathfrak{K}}^{\prime} \otimes 1+\mathscr{U}^{-2} \otimes \widehat{\mathfrak{K}}^{\prime} .
\end{aligned}
$$

\footnotetext{
${ }^{5}$ Note that $f_{B C}^{A} f_{D}^{B C}=0$ here, so there is no contribution from the Lie generators.

${ }^{6}$ The braiding factors in (3.11) turn out to be very important for the Yangian. It can easily be seen that without them the coproduct cannot be quasi-cocommutative. This is in contradistinction to the universal enveloping algebra where the braided as well as the unbraided coproduct are quasi-cocommutative.

${ }^{7}$ Note that the scalar product $\widehat{C} \widehat{\mathfrak{C}}^{\prime}-\frac{1}{2} \mathfrak{P} \widehat{\mathfrak{K}}^{\prime}-\frac{1}{2} \mathfrak{K} \widehat{\mathfrak{P}}^{\prime}=\widehat{C} \widehat{\mathfrak{C}}-\frac{1}{2} \mathfrak{P} \widehat{\mathfrak{K}}-\frac{1}{2} \mathfrak{K} \widehat{\mathfrak{P}}$ is unaffected by the redefinition.
} 


$$
\begin{aligned}
& \Delta \widehat{\Re}^{a}{ }_{b}=\widehat{\mathfrak{R}}^{a}{ }_{b} \otimes 1+1 \otimes \widehat{\mathfrak{R}}^{a}{ }_{b} \\
& +\frac{1}{2} \mathfrak{R}_{c}^{a} \otimes \mathfrak{R}^{c}{ }_{b}-\frac{1}{2} \mathfrak{R}^{c}{ }_{b} \otimes \mathfrak{R}^{a}{ }_{c} \\
& -\frac{1}{2} \mathfrak{S}^{a} \gamma \mathscr{U}^{+1} \otimes \mathfrak{Q}_{b}^{\gamma}-\frac{1}{2} \mathfrak{Q}_{b}^{\gamma} \mathscr{U}^{-1} \otimes \mathfrak{S}^{a} \gamma \\
& +\frac{1}{4} \delta_{b}^{a} \mathfrak{S}^{d} \gamma_{\mathcal{U}^{+1}} \otimes \mathfrak{Q}^{\gamma}{ }_{d}+\frac{1}{4} \delta_{b}^{a} \mathfrak{Q}^{\gamma}{ }_{d} \mathscr{U}^{-1} \otimes \mathfrak{S}^{d}{ }_{\gamma}, \\
& \Delta \widehat{\mathfrak{L}}_{\beta}^{\alpha}=\widehat{\mathfrak{L}}_{\beta}^{\alpha} \otimes 1+1 \otimes \widehat{\mathfrak{L}}_{\beta}^{\alpha} \\
& -\frac{1}{2} \mathfrak{L}^{\alpha}{ }_{\gamma} \otimes \mathfrak{L}^{\gamma}{ }_{\beta}+\frac{1}{2} \mathfrak{L}^{\gamma}{ }_{\beta} \otimes \mathfrak{L}^{\alpha}{ }_{\gamma} \\
& +\frac{1}{2} \mathfrak{Q}^{\alpha}{ }_{c} \mathscr{U}^{-1} \otimes \mathfrak{S}^{c}{ }_{\beta}+\frac{1}{2} \mathfrak{S}^{c}{ }_{\beta} \mathscr{U}^{+1} \otimes \mathfrak{Q}^{\alpha}{ }_{c} \\
& -\frac{1}{4} \delta_{\beta}^{\alpha} \mathfrak{Q}^{\delta}{ }_{c} \mathscr{U}^{-1} \otimes \mathfrak{S}^{c}{ }_{\delta}-\frac{1}{4} \delta_{\beta}^{\alpha} \mathfrak{S}^{c}{ }_{\delta} \mathscr{U}^{+1} \otimes \mathfrak{Q}^{\delta}{ }_{c}, \\
& \Delta \widehat{\mathfrak{Q}}^{\alpha}{ }_{b}=\widehat{\mathfrak{Q}}^{\alpha}{ }_{b} \otimes 1+\mathscr{U}^{+1} \otimes \widehat{\mathfrak{Q}}^{\alpha}{ }_{b} \\
& -\frac{1}{2} \mathfrak{L}^{\alpha}{ }_{\gamma} \mathscr{U}^{+1} \otimes \mathfrak{Q}_{b}^{\gamma}+\frac{1}{2} \mathfrak{Q}_{b}^{\gamma} \otimes \mathfrak{L}^{\alpha}{ }_{\gamma} \\
& -\frac{1}{2} \mathfrak{R}^{c}{ }_{b} \mathscr{U}^{+1} \otimes \mathfrak{Q}^{\alpha}{ }_{c}+\frac{1}{2} \mathfrak{Q}^{\alpha}{ }_{c} \otimes \mathfrak{R}^{c}{ }_{b} \\
& -\frac{1}{2} \mathfrak{C} \mathscr{U}^{+1} \otimes \mathfrak{Q}^{\alpha}{ }_{b}+\frac{1}{2} \mathfrak{Q}^{\alpha}{ }_{b} \otimes \mathfrak{C} \\
& +\frac{1}{2} \varepsilon^{\alpha \gamma} \varepsilon_{b d} \mathfrak{P} \mathscr{U}^{-1} \otimes \mathfrak{S}^{d}{ }_{\gamma}-\frac{1}{2} \varepsilon^{\alpha \gamma} \varepsilon_{b d} \mathfrak{S}^{d}{ }_{\gamma} \mathscr{U}^{+2} \otimes \mathfrak{P}, \\
& \Delta \widehat{\mathfrak{S}}^{a}{ }_{\beta}=\widehat{\mathfrak{S}}^{a}{ }_{\beta} \otimes 1+\mathscr{U}^{-1} \otimes \widehat{\mathfrak{S}}^{a}{ }_{\beta} \\
& +\frac{1}{2} \mathfrak{R}^{a}{ }_{c} \mathscr{U}^{-1} \otimes \mathfrak{S}^{c}{ }_{\beta}-\frac{1}{2} \mathfrak{S}^{c}{ }_{\beta} \otimes \mathfrak{R}^{a}{ }_{c} \\
& +\frac{1}{2} \mathfrak{L}_{\beta}^{\gamma} \mathscr{U}^{-1} \otimes \mathfrak{S}^{a}{ }_{\gamma}-\frac{1}{2} \mathfrak{S}^{a}{ }_{\gamma} \otimes \mathfrak{L}^{\gamma}{ }_{\beta} \\
& +\frac{1}{2} \mathfrak{C} \mathscr{U}^{-1} \otimes \mathfrak{S}^{a}{ }_{\beta}-\frac{1}{2} \mathfrak{S}^{a}{ }_{\beta} \otimes \mathfrak{C} \\
& -\frac{1}{2} \varepsilon^{a c} \varepsilon_{\beta \delta} \mathfrak{K} \mathscr{U}^{+1} \otimes \mathfrak{Q}^{\delta}{ }_{c}+\frac{1}{2} \varepsilon^{a c} \varepsilon_{\beta \delta} \mathfrak{Q}^{\delta}{ }_{c} \mathscr{U}^{-2} \otimes \mathfrak{K}, \\
& \Delta \widehat{\mathfrak{C}}=\widehat{\mathfrak{C}} \otimes 1+1 \otimes \widehat{\mathfrak{C}} \\
& +\mathfrak{P} \mathscr{U}^{-2} \otimes \mathfrak{K}-\mathfrak{K} \mathscr{U}^{+2} \otimes \mathfrak{P}, \\
& \Delta \widehat{\mathfrak{P}}=\widehat{\mathfrak{P}} \otimes 1+\mathscr{U}^{+2} \otimes \widehat{\mathfrak{P}} \\
& -\mathfrak{C} \mathscr{U}^{+2} \otimes \mathfrak{P}+\mathfrak{P} \otimes \mathfrak{C}, \\
& \Delta \widehat{\mathfrak{K}}=\widehat{\mathfrak{K}} \otimes 1+\mathscr{U}^{-2} \otimes \widehat{\mathfrak{K}} \\
& +\mathfrak{C} \mathscr{U}^{-2} \otimes \mathfrak{K}-\mathfrak{K} \otimes \mathfrak{C} .
\end{aligned}
$$

Table 2: The coproduct of the Yangian generators in $\mathrm{Y}(\mathfrak{h})$.

The combination $\widehat{\mathfrak{C}}^{\prime}$ is already cocommutative, and in order to make the generators $\widehat{\mathfrak{P}}^{\prime}, \widehat{\mathfrak{K}}^{\prime}$ cocommutative we have to set as above in $(2.9,2.10)$

$$
\widehat{\mathfrak{P}}^{\prime}=i g u_{\mathfrak{P}} \mathfrak{P}, \quad \widehat{\mathfrak{K}}^{\prime}=i g u_{\mathfrak{K}} \mathfrak{K}
$$

with two universal constants $u_{\mathfrak{P}}$ and $u_{\mathfrak{K}}$. With this choice, $\widehat{\mathfrak{C}}, \widehat{\mathfrak{P}}, \widehat{\mathfrak{K}}$ also become cocommutative because they differ from $\widehat{\mathfrak{C}}^{\prime}, \widehat{\mathfrak{P}}^{\prime}, \widehat{\mathfrak{K}}^{\prime}$ only by central elements. 
Fundamental Evaluation Representation. For the fundamental evaluation representation we make the ansatz ${ }^{8}$

$$
\widehat{\mathfrak{J}}^{A}|\mathscr{X}\rangle=i g\left(u+u_{0}\right) \mathfrak{J}^{A}|\mathscr{X}\rangle .
$$

By comparison with $(3.13,3.15)$ we can infer that $u$ has to be related to the parameters of the fundamental representation by

$$
u=x^{+}+\frac{1}{x^{+}}-\frac{i}{2 g}=x^{-}+\frac{1}{x^{-}}+\frac{i}{2 g}=\frac{1}{2}\left(x^{+}+x^{-}\right)\left(1+1 / x^{+} x^{-}\right) .
$$

Furthermore $u_{\mathfrak{P}}$ and $u_{\mathfrak{K}}$ in (3.15) have to both coincide with the universal constant $u_{0}=u_{\mathfrak{P}}=u_{\mathfrak{K}} .{ }^{9}$

The eigenvalues of the redefined central elements of the Yangian within the evaluation representation read

$$
\widehat{C}^{\prime}=\frac{i g u}{4 C}+i g u_{0} C, \quad \widehat{P}^{\prime}=i g u_{0} P, \quad \widehat{K}^{\prime}=i g u_{0} K .
$$

As an aside we also state the eigenvalue of the quadratic combination

$$
C \widehat{C}-\frac{1}{2} P \widehat{K}-\frac{1}{2} K \widehat{P}=C \widehat{C}^{\prime}-\frac{1}{2} P \widehat{K}^{\prime}-\frac{1}{2} K \widehat{P}^{\prime}=\frac{1}{4} i g\left(u+u_{0}\right) .
$$

Fundamental S-Matrix. Using the coproducts in Tab. 2 we have confirmed that the S-matrix is also invariant under all of the Yangian generators

$$
\left[\Delta \widehat{\mathfrak{J}}^{A}, \mathscr{S}\right]=0
$$

We have used a computer algebra system to evaluate the action of the Yangian generators and the S-matrix. ${ }^{10}$ To show invariance requires heavy use of the identity (2.15). Superficially it is very surprising to find all these additional symmetries of the S-matrix. The deeper reason however should be that the coproduct is quasi-cocommutative. We have thus proved quasi-cocommutativity when acting on fundamental representations.

It is interesting to see that the S-matrix is based on standard evaluation representations of the Yangian. Nevertheless, it is not a function of the difference of spectral parameters. This unusual property traces back to the link between the spectral parameter $u$ and the $\mathfrak{h}$-representation parameters $x^{ \pm}$in (3.17). The latter is again related to the braiding in the coproduct (3.11).

As our S-matrix is equivalent [17] to Shastry's R-matrix, our Yangian is presumably an extension of the $\mathfrak{s u}(2) \times \mathfrak{s u}(2)$ Yangian symmetry of the Hubbard model found in [61].

\section{Conclusions and Outlook}

In this note we have reviewed the construction of the S-matrix with centrally extended $\mathfrak{s u}(2 \mid 2)$ symmetry that appears in the context of the planar AdS/CFT correspondence and the one-dimensional Hubbard model. We have furthermore shown that the S-matrix has an additional Yangian symmetry whose Hopf algebra structure we have presented. This Yangian is not quite a standard Yangian, but its coproduct needs to be braided in order to be quasi-cocommutative. This fact is

\footnotetext{
${ }^{8}$ We believe, but we have not verified that this is compatible with the Serre relations (3.2).

${ }^{9}$ It is conceivable that a further consistency requirement fixes the value of $u_{0}$, presumably to zero.

${ }^{10}$ We have also confirmed the invariance of the singlet state found in [13].
} 
intimately related to the existence of a triplet of central charges with non-trivial coproduct and leads to the wealth of unusual features of the S-matrix.

In connection to the Yangian there are many points left to be clarified. Most importantly the representation theory needs to be understood. Which representations of $\mathfrak{h}$ lift to evaluation representations of $\mathrm{Y}(\mathfrak{h})$ ? At what values of the spectral parameters do their tensor products become reducible? This information could be used to prove that the coproduct is quasi-cocommutative. Also the Yang-Baxter equation for the S-matrix should follow straightforwardly. It might also give some further understanding of bound states [62, 63].

Then it would be highly desirable to construct a universal R-matrix for this Yangian and show that it is quasi-triangular. This would put large parts of the integrable structure for arbitrary representations of this algebra on solid ground much like for the case of generic simple Lie algebras.

Some further interesting questions include: Is this Yangian the unique quasi-cocommutative Hopf algebra based on $\mathfrak{h}$ ? Does the double Yangian [50] exist and what is its structure? Can the $\mathfrak{s l}(2)$ automorphism of the algebra be included at the Yangian level such that the coproduct is quasi-cocommutative? What would the representations be in this case?

Acknowledgements. I thank C. Callan, D. Erkal, A. Kleinschmidt, P. Koroteev, N. MacKay, M. Mariño, T. McLoughlin, J. Plefka, F. Spill and B. Zwiebel for interesting discussions.

\section{References}

[1] H. Bethe, Z. Phys. 71, 205 (1931).

[2] W. Heisenberg, Z. Phys. 49, 619 (1928).

[3] J. M. Maldacena, Adv. Theor. Math. Phys. 2, 231 (1998), hep-th/9711200.

[4] J. A. Minahan and K. Zarembo, JHEP 0303, 013 (2003), hep-th/ 0212208.

[5] N. Beisert, C. Kristjansen and M. Staudacher, Nucl. Phys. B664, 131 (2003), hep-th / 0303060.

[6] N. Beisert and M. Staudacher, Nucl. Phys. B670, 439 (2003), hep-th/ 0307042.

[7] G. Mandal, N. V. Suryanarayana and S. R. Wadia, Phys. Lett. B543, 81 (2002), hep-th/ 0206103.

[8] I. Bena, J. Polchinski and R. Roiban, Phys. Rev. D69, 046002 (2004), hep-th/ 0305116.

[9] N. Beisert and M. Staudacher, Nucl. Phys. B727, 1 (2005), hep-th/ 0504190.

[10] N. Beisert, B. Eden and M. Staudacher, J. Stat. Mech. 07, P01021 (2007), hep-th/ 0610251.

[11] M. Staudacher, JHEP 0505, 054 (2005), hep-th / 0412188.

[12] N. Beisert, Phys. Rept. 405, 1 (2004), hep-th/0 407277.

[13] N. Beisert, hep-th/0511082.

[14] W. Nahm, Nucl. Phys. B135, 149 (1978).

[15] C. Gomez and R. Hernández, JHEP 0611, 021 (2006), hep-th/ 0608029.

[16] J. Plefka, F. Spill and A. Torrielli, Phys. Rev. D74, 066008 (2006), hep-th $/ 0608038$.

[17] N. Beisert, J. Stat. Mech.07, P01017 (2007), nlin.SI / 0610017.

[18] N. Beisert, P. Koroteev and F. Spill, in preparation.

[19] G. Arutyunov, S. Frolov and M. Zamaklar, hep-th/0612229.

[20] M. J. Martins and C. S. Melo, hep-th/0703086.

[21] B. S. Shastry, Phys. Rev. Lett. 56, 2453 (1986).

[22] J. Hubbard, Proc. R. Soc. London A276, 238 (1963).

[23] E. H. Lieb and F. Y. Wu, Phys. Rev. Lett. 20, 1445 (1968).

[24] A. Rej, D. Serban and M. Staudacher, JHEP 0603, 018 (2006), hep-th/ 0512077.

[25] D. Serban and M. Staudacher, JHEP 0406, 001 (2004), hep-th/ 0401057.

[26] G. Arutyunov, S. Frolov and M. Staudacher, JHEP 0410, 016 (2004), hep-th 0406256.

[27] N. Beisert and A. A. Tseytlin, Phys. Lett. B629, 102 (2005), hep-th/ 0509084.

[28] R. Hernández and E. López, JHEP 0607, 004 (2006), hep-th/ 0603204. 
[29] N. Gromov and P. Vieira, hep-th/0703191.

[30] R. A. Janik, Phys. Rev. D73, 086006 (2006), hep-th/ 0603038.

[31] G. Arutyunov and S. Frolov, Phys. Lett. B639, 378 (2006), hep-th/ 0604043.

[32] N. Beisert and T. Klose, J. Stat. Mech. 06, P07006 (2006), hep-th/ 0510124.

[33] N. Beisert, R. Hernández and E. López, JHEP 0611, 070 (2006), hep-th/ 0609044.

[34] J. K. Erickson, G. W. Semenoff and K. Zarembo, Nucl. Phys. B582, 155 (2000), hep-th / 0003055.

[35] D. J. Gross and N. Miljkovic, Phys. Lett. B238, 217 (1990).

[36] A. V. Kotikov and L. N. Lipatov, hep-th/0611204.

[37] A. V. Belitsky, hep-th/0703058.

[38] N. Dorey, D. M. Hofman and J. Maldacena, hep-th/0703104.

[39] K. Sakai and Y. Satoh, hep-th/0703177.

[40] A. Rej, M. Staudacher and S. Zieme, hep-th/0702151.

[41] N. Mann and J. Polchinski, Phys. Rev. D72, 086002 (2005), hep-th/ 0508232.

[42] N. Gromov, V. Kazakov, K. Sakai and P. Vieira, Nucl. Phys. B764, 15 (2007), hep-th/ 0603043.

[43] Z. Bern, M. Czakon, L. J. Dixon, D. A. Kosower and V. A. Smirnov, hep-th/ 0610248.

[44] M. K. Benna, S. Benvenuti, I. R. Klebanov and A. Scardicchio, hep-th/ 0611135.

[45] M. Beccaria, G. F. De Angelis and V. Forini, hep-th/ 0703131.

[46] L. F. Alday, G. Arutyunov, M. K. Benna, B. Eden and I. R. Klebanov, hep-th/ 0702028.

[47] I. Kostov, D. Serban and D. Volin, hep-th/0703031.

[48] J. Maldacena and I. Swanson, hep-th/0612079.

[49] V. G. Drinfel'd, Sov. Math. Dokl. 32, 254 (1985).

[50] V. G. Drinfel'd, J. Sov. Math. 41, 898 (1988).

[51] D. Bernard, Int. J. Mod. Phys. B7, 3517 (1993), hep-th/9211133.

[52] N. J. MacKay, Int. J. Mod. Phys. A20, 7189 (2005), hep-th/ 0409183.

[53] L. Dolan, C. R. Nappi and E. Witten, JHEP 0310, 017 (2003), hep-th/ 0308089.

[54] L. Dolan, C. R. Nappi and E. Witten, hep-th/0401243, in: "Quantum theory and symmetries", ed.: P. C. Argyres et al., World Scientific (2004), Singapore.

[55] M. Hatsuda and K. Yoshida, Int. J. Mod. Phys. A19, 4715 (2004), hep-th / 0407044.

[56] L. Dolan and C. R. Nappi, Nucl. Phys. B717, 361 (2005), hep-th/ 0411020.

[57] A. Agarwal and S. G. Rajeev, Int. J. Mod. Phys. A20, 5453 (2005), hep-th/ 0409180.

[58] N. Berkovits, JHEP 0503, 041 (2005), hep-th/ 0411170.

[59] B. I. Zwiebel, J. Phys. A40, 1141 (2007), hep-th/ 0610283.

[60] N. Beisert and D. Erkal, in preparation.

[61] D. B. Uglov and V. E. Korepin, Phys. Lett. A190, 238 (1994), hep-th/ 9310158.

[62] N. Dorey, J. Phys. A39, 13119 (2006), hep-th/ 0604175.

[63] H.-Y. Chen, N. Dorey and K. Okamura, JHEP 0611, 035 (2006), hep-th/ 0608047. 